\title{
INVESTIGATIONS OF TWO TYPES DISORDER EFFECTS ON ONE- DIMENSIONAL ROAD TRAFFIC
}

\author{
A.K. Daoudia ${ }^{1}$, Y. El Hassouani ${ }^{2}$, A. Benami ${ }^{3}$ \\ ${ }^{I}$ OTEA, Département De Physique, FSTE, BP 509 Boutalamine, Errachidia, Morocco \\ ${ }^{2}$ ESIM, Département De Physique, FSTE, BP 509 Boutalamine, Errachidia, Morocco \\ ${ }^{3}$ OTEA, Département De Physique, FSTE, BP 509 Boutalamine, Errachidia, Morocco
}

\begin{abstract}
We study a simple one lane cellular automaton, based upon the well-known Nagel-Schreckenberg model, and examine the effect of disorder caused by some defects (deceleration sites, temporary stop sites and presence of slow cars) in such a system. We point out the important parameters defining the shape of the fundamental diagram for the NaSch model and compare it to that of one with sites or particles disorder, showing a new mode of interactions between vehicles. Important differences in the flow behavior between these types of defects and their impact on the occurrence of congestions have been established.
\end{abstract}

Keywords: Vehicular Traffic, Cellular Automata, Congestions

\section{INTRODUCTION}

In recent times many highway traffic models formulated in terms of cellular automata (CA) have been studied on one lane [1-7]. For a realistic description of traffic on highways, we must mention in particular the model introduced by Nagel and Schreckenberg, which has been generalized in the past years to develop a CA model of $t$ multi-lane traffic [814]. Furthermore, several attempts have so far been to understand the disorder effect on a system one lane [15-16]. The simulation results have shown that, in the case of particle-disorder, already for small densities, the fast vehicles take on the average free-tow velocity of the slow vehicles, even if only a small fraction of slow vehicles are considered. Recent simulation results of Knospe et al. [13] considered an anticipation effect and found that it reduces the influence of the slow vehicles drastically. In the case of defect-site (or impurity site) several types of these defects have been explored $[17,18]$. That corresponds to a section of road where vehicles are forced to decelerate (works) or stop for a moment before returning (accidents).

Recently, some studies examine the cellular automata traffic flow model, which considers the heterogeneity of vehicle acceleration and the delay probability of vehicles $[19,20]$.

In general, different types of vehicles are present in traffic, and from daily experience it is known that slow vehicles have a strong influence on the system's performance. This is obviously most pronounced in single-lane traffic where passing is not possible, and the slow vehicles dominate the dynamics.

Our model is the NaSch model defined on the 1-D lattices of $\mathrm{N}$ sites with periodic boundary conditions, forming a closed circuit. In this work, we summarize the definition of the single-lane model and present a characterization of defectsites and defect-particle. Simulation results of such systems compared to the systems not containing any disorder and the influence of disorder on its dynamics are presented. Paragraph comes content here. Paragraph comes content here. Paragraph comes content here. Paragraph comes content here.

\section{CHARACTERIZATION OF THE MODEL}

The NaSch model is a CA model [3] which is described as shown in figure 1: on a ring with $\mathrm{L}$ sites every site can either be empty or occupied by one vehicle with velocity $v=0$, $1, . ., v_{\max }$. Let gap be the number of empty sites in front of the car, and $v$ its velocity at time $t$. At each discrete time step the arrangement of $N$ cars is updated in parallel, according to the following rules:

- Acceleration: with regard to the vehicle ahead: $v^{\prime}=\min (v$ +1 ; gap; $\left.v_{\max }\right)$

- Noise (or deceleration): with a probability $p: v^{\prime \prime} \max \left(v^{\prime}-1\right.$; 0 )

- Movement: the car moves v"' sites ahead.

Configuration at time $\mathrm{t}$

\begin{tabular}{|c|c|c|}
\hline$\leftrightarrow^{2}$ & $\cos ^{x}$ & $\Omega^{x} \leftrightarrows \overbrace{}^{\prime \prime}$ \\
\hline
\end{tabular}

Acceleration

\begin{tabular}{|c|c|c|c|}
\hline$\epsilon^{2}{ }^{2}$ & $63^{2}$ & $43^{2} 4 \pi^{1}$ & \\
\hline
\end{tabular}

Deceleration

\begin{tabular}{|c|c|c|}
\hline$\Phi^{\prime}$ & $4 s^{2}$ & $\Leftrightarrow$ \\
\hline
\end{tabular}

Probability $p=1 / 3$

$\left|\oplus^{\circ}\right|\left|\omega^{2}\right| \quad\left|\Theta^{\circ}\right| \Theta^{\prime}|\quad| \longrightarrow$

Movement at time $\mathrm{t}+1$

$\left|\omega^{\circ}\right| \quad|\quad| \omega^{2}\left|\omega^{\circ}\right| \quad\left|\omega^{2}\right| \longrightarrow$

Fig -1: Example of the NaSch rules for vehicles movement 
It is clear that this model is minimal in the sense that all the rules above are necessary to reproduce the basic characteristics of real traffic. However, additional rules are also needed to capture more complex situations. Note that changing the order of these rules changes the properties of the model.

As regards the real traffic data simple calibration can be done. The length of the cell is about $7.5 \mathrm{~m}$, interpreted as the vehicle length plus the distance separating two successive vehicles in a traffic jam. The maximum velocity $v_{\max }=5$ corresponds to a desired speed of $130 \mathrm{~km} / \mathrm{h}$, and the duration of a simulation step refers to approximately 1 second. The model of NaSch may be considered a probabilistic CA (or stochastic). In the particular case where $v_{\max }=1$, the properties of stationary states can be obtained exactly and deterministic limit of the model is then equivalent to the rule CA-184 in ratings Wolfram [2]. However, for the most interesting cases where $v_{\max }>1$, we can only rely on MonteCarlo simulations or approximate methods.

In this work we first present a characterization of NaSch model starting with the description of the effect of density and probability of deceleration in a one dimensional lattice.

One good way to visualize traffic and traffic flow is to use the space-time diagrams giving the time evolution of $\mathrm{NaSch}$ model. The figure 2 and 3 shows that there is no congestions phenomenon to sufficiently low densities but spontaneous fluctuations at high densities lead to it. The results of two simulations of NaSch model are obtained for low density ( $\square=0.09$ ) and relatively high density $(\square=0.2)$ and the parameters $v_{\max }=5$ and $p=0.25$.

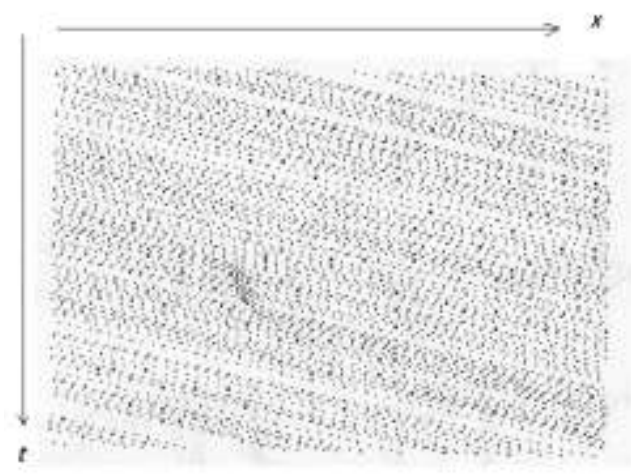

Fig-2: Space-time diagram of NaSch model with $v_{\max }=5, p$ $=0.25$ and a subcritical density $\square=0.09$.

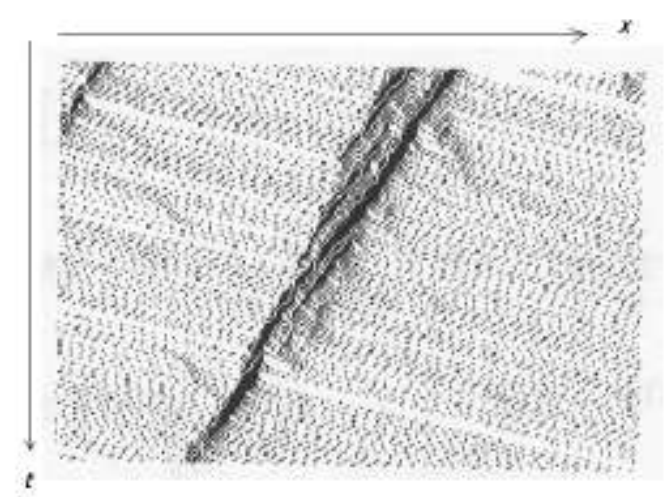

Fig-3: Space-time diagram of NaSch model with $v_{\max }=5, p$ $=0.25$ and a supercritical density $\square=0.2$.
Furthermore, examination of the rear edge of congestion on space-time diagrams proves that this front back on the road (Fig. 4). Note however that even without measures, it was possible to predict the necessity of moving backward of congestion fronts indeed, as a vehicle interacts with the one placed immediately before him, any observable macroscopic phenomenon propagating can do it in opposite direction of movement of particles.

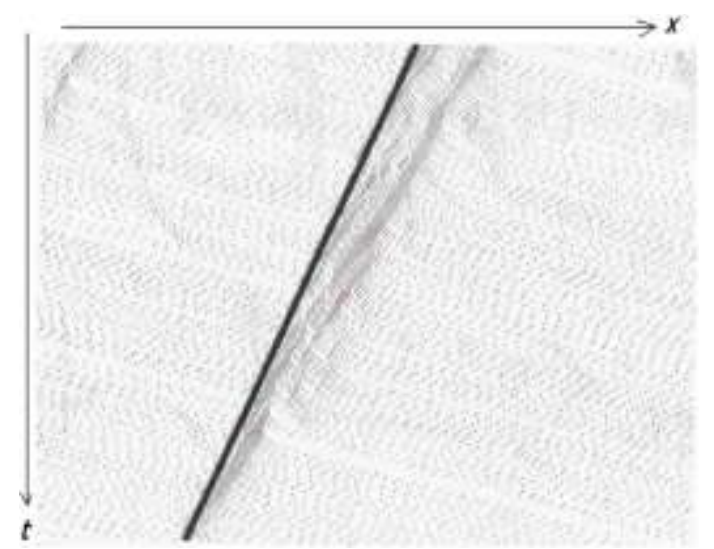

Fig-4: Space-time diagram of NaSch model with $v_{\max }=5, p$ $=0.4$ and a supercritical density $\square=0.2$. The added line indicates the front of traffic jams.

In supercritical regime, observation highways showed that traffic is divided into two zones, congested areas and fluid zones. It showed that the density in the fluid zones (that is to say between two jams) was constant and does not depend on the average density of the entire road. Other conditions in reality as the average type of vehicle and the topography of the road, criteria modelled in their entirety by the value of the probability of deceleration $\mathrm{p}$, is a phenomenon known as self-organized criticality, term that expresses the trend in traffic to be divided into congested areas and exactly critical areas.

Moreover, it is interesting to see how the microscopic structure of the stationary states depends on the probability of deceleration $\mathrm{p}$ (Fig. 5). It is possible to accurately determine the fundamental diagram for the model in its deterministic limit $p=0$, which simply corresponds to the removal of the third rule of cellular automata (which introduced a random factor). For $p=0$, we show that for low densities, vehicles have a speed uniformly equal to $v_{\max }$, and therefore they never see other vehicles in front of them [9]. In this limit, the density is then called critical and the flow is maximal, it reached the capacity. This is no possible for large densities but vehicles keep their tendency to minimize their progress. In summary, the speed is $v_{\max }$ in free regime. We conclude that for $p=0$, the formation of spontaneous congestions does not exist. 


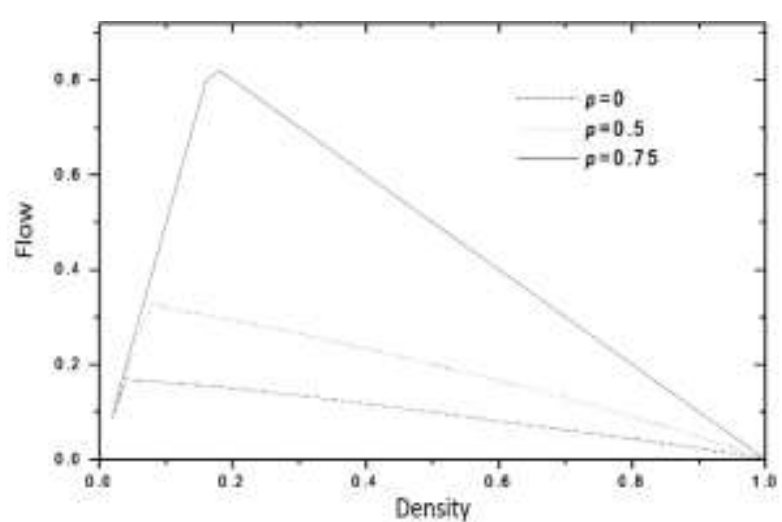

Fig-5: Fundamental diagram for $v_{\max }=5$ and different values of the probability of deceleration: $p=0,0.05$ and 0.75 .

The case $p=1$ is slightly different. It shows that there are metastable states with a finite flow for and $v_{\max }>1$. For 0 $<p<1$, the microscopic structure of the stationary state is located between the two limiting cases $\left(p=0\right.$ and $v_{\max }$ is any) and ( $v_{\max }=1$ and $p$ arbitrary).

Now, we present a few applications of the NaSch model through description of the profile of disorder in a one dimensional lattice which can be caused by decelerate sites, temporary stop sites or by the presence of slow vehicles.

\section{DISORDER EFFECTS: DISCUSSIONS AND RESULTS}

A fundamental problem of the theory of vehicular traffic on a one lane road is the formation of congestions that may form spontaneously as well that because of accident, works and the presence of slow moving vehicles or other possible discomfort on the road. Several types of these obstacles have been studied, especially defect-sites and particledisorder (slow vehicles).

\subsection{Deceleration Sites}

We studied the impact of these types of defects on traffic in closed circuits using the model of NaSch, like several authors. The disorder particles may have a low maximum velocity and are not localized in space; in contrast, the defect-sites have a precise position and may be sites where the probability of deceleration of vehicles is increased or sites where vehicles must wait a while before returning. All these types of disorder induce phase separation between the regions of low and high densities.

We simulated the NaSch model with $v_{\max }=5$, and a probability of deceleration $p=0.4$ for a system having a number of defect-site equal to $v_{\max }$ and where the deceleration probability of vehicles $p_{\mathrm{d}}$ is greater than $\mathrm{p}$ applied in the rest of the system sites.

The average flow of such a system as shown in figure 6 has the same values as a homogeneous system (not containing defect-site) for extremely low and high densities. For intermediate densities, the flow stabilizes around a constant value.

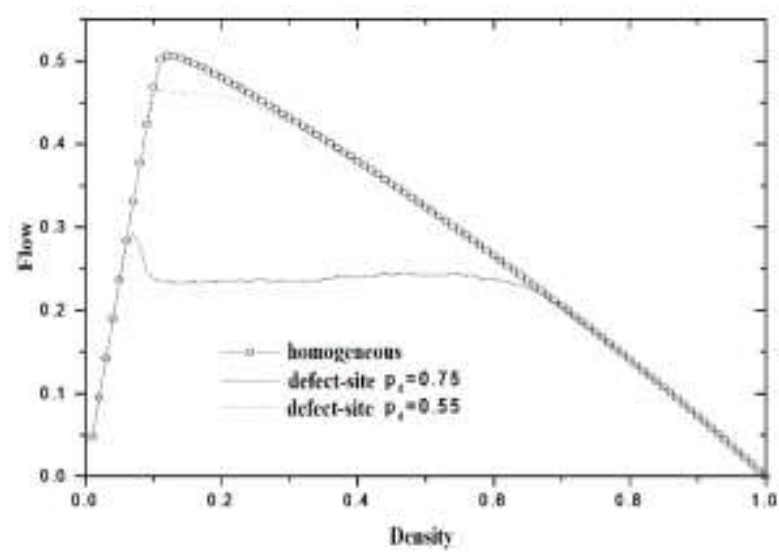

Fig-6: Fundamental diagrams of a homogeneous system compared to its corresponding containing $v_{\max }$ defect-sites with $p_{\mathrm{d}}=0.55$ and $p_{\mathrm{d}}=0.75$. The deceleration probability in the rest of the circuit being $p=0.4$.

For a system with a single defect-site, the same phenomenon is observed. Knospe et al. [13] explain this behavior in that for the low and high densities phases, only local heterogeneity exist in the vicinity of the defect-sites, this is why the flow is similar to that of a system without defects. In contrast, for intermediate values of density, phase separation is observed between low and high densities.

Moreover, we note that if the probability of deceleration is elevated, the flow is affected by a drastic reduction.

\subsection{Temporary Stop Sites}

We then interested in studying a system with a defect-site where vehicles are forced to wait for a time before returning. For $t=2$ and $t=3$ waiting times (instead of $t=1$ in the rest of the system's sites), a behavior similar to that observed in the case of defect-site of the first type was observed as shown in figure 7. Obviously, if the waiting time is large the flow is reduced.

We can notice that the effect of this type of disorder on the average flow is similar to that caused by deceleration-sites in the sense that for intermediate values of density there is a phase separation between low and high densities.

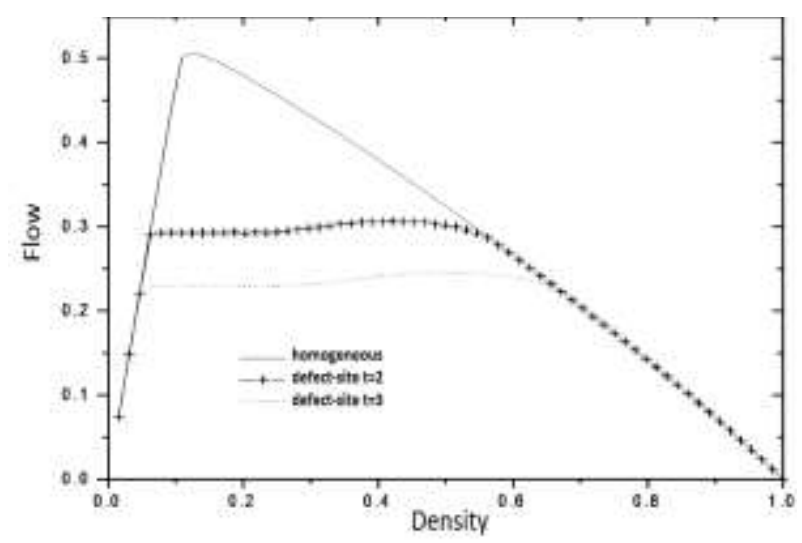

Fig-7: Average flux in function of the density in the Nasch model for a homogeneous system and one system with a defect-site where vehicles mark a stop during $t=2$ (or $t=3$ ) and $v_{\max }=5$ and $p=0.4$. 


\subsection{Temporary Stop Sites}

We then studied the systems which we introduced $N_{s}$ disorder particles (slow vehicles). Knospe and al. [13] showed that a single of these particles is sufficient to produce, for low densities, phase separation of the stationary states. These statements consist of large congestion following the slow vehicle and a large distance separating the latter from its predecessor.

We examined a system with $N_{s}=1,5$ and 10 slow vehicles with $v_{\text {max }}^{\text {truck }}=3$. The probability of deceleration is $p=0.4$ for all vehicles. The results of simulations of such a system compared to a corresponding homogeneous system are given in Figure 8. Overtaking is not possible in a single lane road, it is clear that slow vehicles dominate the flows at low densities and congestions form in their wake. For larger densities than $\square_{\text {jmax }}$ (the value corresponding to the maximum flow), slow vehicles still are not sufficient distances before them. Thus the average flow is limited by the number of unoccupied sites. Therefore, there is obtained the same flow to a homogeneous system of fast vehicles and an inhomogeneous system. For very low densities, the presence of one or more slow vehicles in the system greatly increases the risk of accidents since the latter is directly related to stationary vehicles. In addition, increasing the number of slow vehicles Ns does not increase the disorder in the system for very low densities.

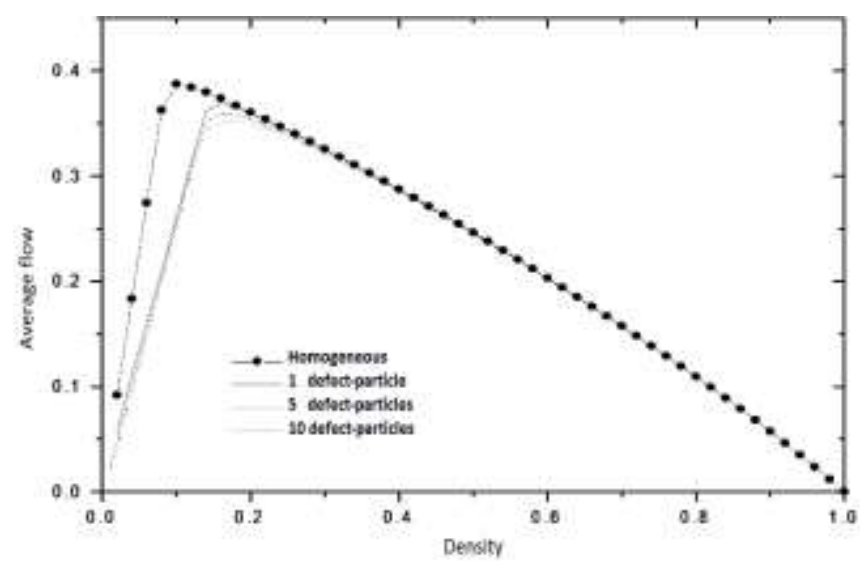

Fig-8: Fundamental diagram for a homogeneous system compared with its corresponding containing 1,5 or 10 defect particles, maximum velocities being $v_{\max }=5$ and $v_{\max }^{\text {truck }}=3$, and $p=0.4$

\section{CONCLUSION}

We have presented in this paper an extension of a deterministic cellular automaton model of NaSch to traffic flow in a single roadway with two types of defects (defectsites and defect-particles). Our simulations have confirmed that both types of disorder can change the microscopic properties of the model and induced a phase separation of states. A systematic comparison between this model and its corresponding without defects was made. These defects disrupt the system in the sense that they involve a reduction in the maximum flow and greatly increase the occurrence of congestions at very low densities. On the other hand, the investigations showed that the effect of deceleration-sites and temporary stop sites on the flow is comparable in the sense that even induces the similar phase separation. By against defect-particles have an impact on the flow primarily to low densities.

\section{REFERENCES}

[1]. N.H. Gartner and N.H.M. Wilson (eds) (1987) Transportation and TrafficTheory (New york: Elsevier).

[2]. S. Wolfram, Theory and Application of Cellular Automata, (World Scientific, Singapore, (1986).

[3]. K. Nagel and M. Schreckenberg, J.Physique I, 2, 2221 (1992).

[4]. M. Bengrine, A. Benyoussef, Ez-Zahraouy, J.Krug, M.Loulidi and F. Mhirech, J. Phys. A: Math. Gen. 32 (1999) 2527-2540

[5]. D. Chowdhury, L. Santen and A. Schadschneider; Physics Reports 329,199 (2000).

[6]. A. Benyoussef, H. Chakib and H. Ez-Zahraouy, Euro. phys. J. B 8, 275(1999).

[7]. M. Fukui and Y. Ishibashi, J.Phys. Soc. Jpn. 62 (1993)

[8]. D. Chowdhury, D.E. Wolf and M. Schreckenberg, Physica A 235, 417(1997).

[9]. A. Benyoussef, N. Boccara, H. Chakib and H. EzZahraouy, Chinese Journal of physics, Vol. 39, NO.5, Oct (2001).

[10]. A.K. Daoudia, N. Moussa, Chinese Journal of Physics 40 (5), 484-489(2002).

[11]. A.K. Daoudia, N. Moussa Chinese journal of physics 41 (6) 671-681(2003).

[12]. N. Moussa and A.K. Daoudia, Eur. Phys. J. B 31, 413420 (2003).

[13]. W. Knospe, L. Santen, A. Schadschneider and M. Schreckenberg, PhysicaA 265, 614 (1999).

[14]. T. Nagatani, J. Phys. A: Math.Gen. 26, (1993) L781.

[15]. A. Benyoussef, N. Boccara, H. Chakib and H. EzZahraouy, Chinese Journal of physics, Vol. 39, NO.5, Oct (2001).

[16]. R. Marzoug, H. Ez-Zahraouy, and A. Benyoussef, Int. J. Mod. Phys. C 26, 1550007 (2015).

[17]. N.Boccara, H. Fuks and Q. Zeng, J. Phys. A: Math. Gen. 30 (1997) 3329-3332.

[18]. N. Moussa, Phys. Rev. E 68, 036127 (2003).

[19]. Qi-Lang Li, S.C. Wong, Jie Min, Shuo Tian, BingHong Wang, Physica A: Statistical Mechanics and its Applications, 456, 128-134 (2016).

[20]. Da Yanga, Xiaoping Qiua, Dan Yua, RuoxiaoSuna, Yun Pu, Physica A: Statistical Mechanics and its Applications, 424, 62-72(2015). 


\section{BIOGRAPHIES}

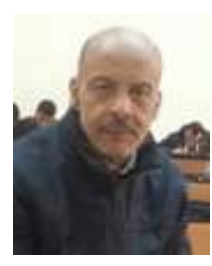

Abdelkarim Daoudia, Phd of the University of Provence (France). Interested research: Condenser Matter, Vehicular traffic, Statistical physics.

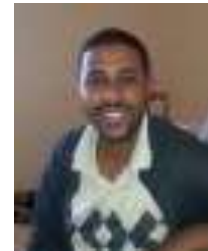

Youssef El Hassouani, Phdof the University of Lille 1 (France) and University Mohammed Premier Oujda (Morocco). Interested research:Phononics photonics crystals, Solar Photovoltaic Technology.

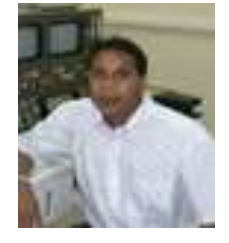

Abdellah Benami, $\mathrm{PhD}$ degree in science and engineering of materials from the National Autonomous University of Mexico (UNAM) in 2008. In 2008 he was granted the Alfonso Caso Medal as a recognition of his performance in $\mathrm{PhD}$ from UNAM. Researcher at the department of physics, Faculty of Science and Technology-Errachidia (Morocco). Mains interests: plasmonics, nanotechnology, metallic and semiconducting nanoparticles and renewable energy. 